\title{
Wind Energy Development and Technology in the World: A Brief Overview
}

\author{
José Luis Elizondo, Marco Rivera, and Patrick Wheeler
}

\begin{abstract}
A brief overview of wind energy 2018 market status with short- and long-term projections will be presented, as well as the wind turbine technologies and market share. Furthermore, clean energy goals from the governments of Denmark, Mexico and Chile will be mentioned, highlighting the world's ongoing transition to a low emissions society.
\end{abstract}

Keywords-Development, Renewable Energy, Technology, Wind Energy.

\section{INTRODUCCIÓN}

Haciendo una retrospectiva de veintidós años, se pueden enumerar una serie de avances para denotar el progreso de la transición del uso de energías fósiles al uso de las energías renovables para paso a paso acercarnos cada vez más al objetivo de ser una sociedad de bajas emisiones. Enfocando el análisis, de dicho progreso, específicamente al uso de la Energía Eólica (EE), se pueden listar los siguientes hitos [1]:

- 1997 Protocolo de Kioto

- 2005 Capacidad Mundial de EE 50 GW

- 2008 Capacidad Mundial de EE 115 GW

- 2010 Precio de Subasta de EE 80 USD/MWh

- 2015 Acuerdo de París

- 2018 Capacidad Mundial de EE 590 GW con Precio de Subasta 21 USD/MWh

Los cuales junto con el resto de las energías renovables, como parte de la llamada transformación energética, han ido aportando beneficios clave como costos de energía renovable más bajos, aumento a la posibilidad de acceso a la energía, reducción de emisiones y contaminantes al aire, incremento del bienestar social y crecimiento económico [1].

Uno de los indicadores más impactantes del crecimiento en capacidad de generación de energía por cada turbina eólica es el diámetro del área barrida por las aspas como se muestra en la Fig. 1, pasando de 15 m en 1985 a 164 m en 2017 [2], con instalación de modelos de $174 \mathrm{~m}$ en el 2023 [3] y proyección a corto plazo de desarrollos de prototipos con 220 $\mathrm{m}$ de diámetro [4].

Sin embargo, la capacidad de generación no solo depende del tamaño y diseño de las aspas, sino también del tipo de tecnología que se utilice en el generador eléctrico y en los controladores electrónicos de potencia, con lo cual es posible captar más energía aún y con el mismo diámetro de área de barrido de las aspas [5].

J.L. Elizondo is with Tecnológico de Monterrey, Campus Monterrey, México, e-mail: jl.elizondo.carrales@ieee.org.

M. Rivera is with Universidad de Talca, Campus Curicó, Chile, e-mail: marcoriv@utalca.cl.

P. Wheeler is with University of Nottingham, Main Campus, U.K., e-mail: patrick.wheeler@nottingham.ac.uk.

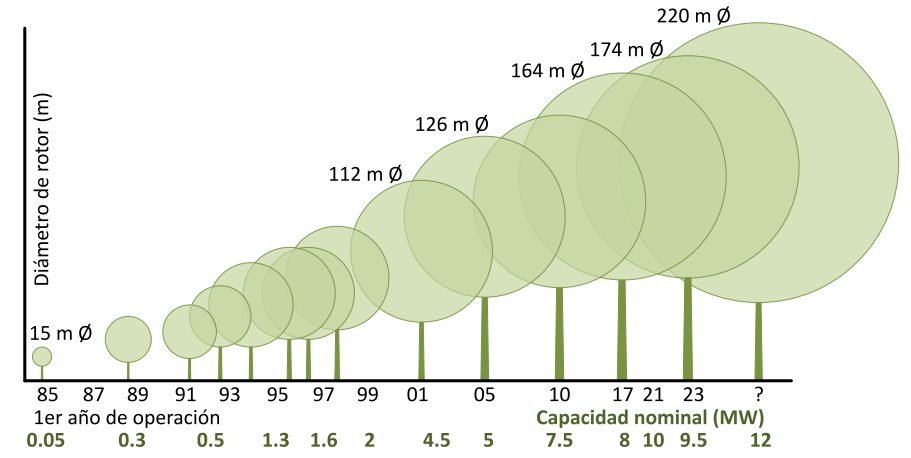

Figura 1. Crecimiento en capacidad y diámetro de rotor de turbinas eólicas de 1985 a 2023 [2], [3], [4], [5].

Para analizar el desarrollo histórico, presente y proyección a futuro del uso de los diferentes tipos de tecnologías en la EE, este artículo se encuentra organizado de la siguiente manera. Primero se expone el estatus del mercado actual reportado por el Consejo Global de Energía Eólica (GWEC por sus siglas en Inglés) para posteriormente presentar las proyecciones a corto y largo plazo del mismo consejo. Después se presenta la información pública disponible de diferentes firmas de análisis de mercado para presentar el uso actual de las diferentes tecnologías de turbinas eólicas y su cuota aproximada del mercado. Luego se mencionan las metas de energías limpias de los gobiernos de Dinamarca (punta de lanza de los países de la Unión Europea en vías de descarbonización e independencia de los combustibles fósiles), México y Chile (parte fundamental del desarrollo de la energía eólica en Latinoamérica). Y para finalizar, una reflexión respecto a la transformación de la industria de los combustibles fósiles a industrias de la energía.

\section{ESTATUS DEL MERCADO 2018}

La capacidad instalada acumulada total en el mundo ha crecido a razón de $20.7 \%$ en promedio en los últimos 18 años, pasando de $24 \mathrm{GW}$ en el año 2001 instalados costa adentro, hasta llegar a $591 \mathrm{GW}$ en el año 2018, de los cuales $23 \mathrm{GW}$ están instalados costa afuera [6]. La instalación de nueva capacidad muestra una tendencia a la alza pasando por varios altibajos como se muestra en la Fig. 2, donde en el año 2013 hubo una reducción del $20 \%$ con respecto al 2012 y en el 2016 de $14 \%$ con respecto a 2015, donde este último año ha sido el mejor en la historia de la energía eólica con el récord de mayor capacidad instalada nueva. De la misma Fig. 2 se puede notar que si bien los últimos tres años se ha presentado una ligera reducción en las nuevas instalaciones, éstas a su vez han mostrado un aumento en la instalación de capacidad costa afuera con $4.5 \mathrm{GW}$ en los años 2017 y 2018. Aún y con 


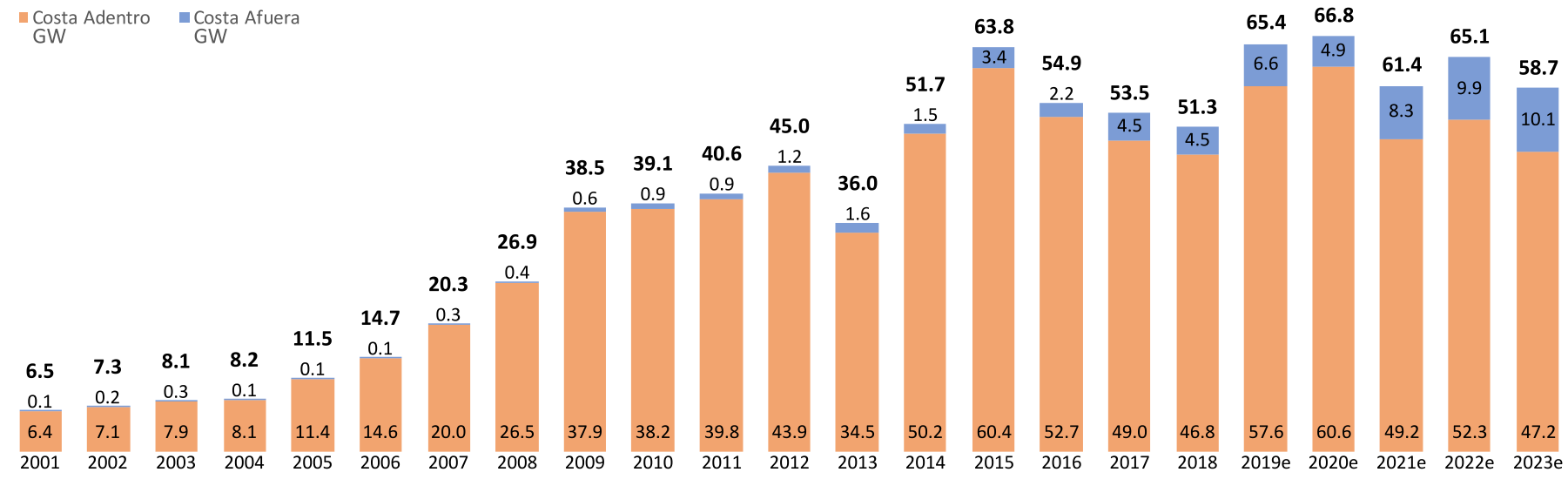

Figura 2. Nuevas instalaciones de capacidad, histórico de 2001 a 2018 y proyección de 2019 a 2023 [6].

este comportamiento, la tendencia sigue siendo a la alza para las nuevas instalaciones en proyecciones futuras. Lo anterior con base en la reducción en los costos nivelados de la energía (LCOE por su siglas en Inglés) eólica reportados en [6], que en el año 2010 estaban en poco más de 80 USD/MWh para costa adentro y en poco menos de $180 \mathrm{USD} / \mathrm{MWh}$ para costa afuera, y en 2018 se encontraban en poco más de 50 USD/MWh y en poco menos de $120 \mathrm{USD} / \mathrm{MWh}$ respectivamente.

El país con la mayor capacidad instalada total acumulada costa adentro al cierre del año 2018 es China con 36\%, seguido por Estados Unidos con $17 \%$ y en tercer lugar Alemania con $9 \%$. El único país de América Latina que figura entre los primeros 10 es Brasil, en el séptimo lugar con $3 \%$ del total de $568 \mathrm{GW}$ [6]. El país con la mayor capacidad instalada total acumulada costa afuera al cierre del año 2018 es el Reino Unido con $34 \%$, seguido por Alemania con $28 \%$ y en tercer lugar China con $20 \%$ del total de $23.1 \mathrm{GW}$ [6]. En cuanto a las estadísticas de las nuevas instalaciones en el año 2018, los tres primeros lugares costa adentro se mantienen igual que con la capacidad instalada total acumulada, China con $45 \%$, EE.UU. con $16 \%$ y Alemania con $5 \%$, pero entre los primeros 10 ahora figuran dos países de América Latina, Brasil en el quinto lugar con $3 \%$ y México en el séptimo con $2 \%$ del total de $46.8 \mathrm{GW}$ [6]. Los tres primeros lugares costa afuera son China con $40 \%$, Reino Unido con $29 \%$ y Alemania con $22 \%$ del total de $4.5 \mathrm{GW}$ [6].

Los países de América Latina que durante los últimos cuatro años han estado contribuyendo fuertemente instalando nueva capacidad de energía eólica son Brasil, México y Chile, en orden de contribución, con la inclusión de Argentina en el año 2018 que llegó a superar la instalación que hizo México en el año 2017 y se colocó en tercer lugar en 2018 donde Brasil contribuyó con 1,939 MW, México 929 MW, Argentina 494 MW, Chile 204 MW y el resto de América Latina con 220 MW [6].

\section{PRoyecciones a CORTO y LARgo Plazo}

Como se aprecia en la Fig. 2, de acuerdo con lo reportado en [6], para los años 2019, 2020 y 2022, se esperan niveles de instalaciones de nueva capacidad que superen el récord del año 2015, donde de 2019 a 2023 se esperan instalaciones con un mínimo de 58.7 GW en 2023 y un máximo de 66.8 GW en 2020. Durante esta proyección a 5 años la participación de China se espera que sea por encima del $30 \%$, la de Europa alrededor del $20 \%$, una disminución de la participación de América del Norte en el año 2021 de alrededor de $20 \%$ a alrededor de $10 \%$, así como un aumento de las instalaciones en el resto de Asia en los años 2021 y 2022 de alrededor de $10 \%$ a alrededor del $15 \%$, y a partir de 2021 un aumento en las instalaciones de aerogeneradores costa afuera de alrededor de $9 \%$ a alrededor del $15 \%$ con una participación de América Latina y África, ambas manteniéndose por debajo del $10 \%$.

De acuerdo con la proyección a 2050 en [7] de la capacidad instalada total en el mundo, considerando que la razón de crecimiento de 2015 a 2018 permaneciera igual hasta el 2020, entonces la realidad se ubicaría entre el escenario 450 de la Agencia Internacional de Energía (IEA por sus siglas en Inglés) y el escenario moderado del GWEC, con $696.7 \mathrm{GW}$ en 2020 y $3,754.2 \mathrm{GW}$ en 2050. Estos datos representarían que alrededor del $7.5 \%$ del total de la demanda de electricidad en el mundo para 2020 y alrededor del $25 \%$ para el 2050 sería suministrada con energía eólica.

\section{TeCnOlogías EN AEROGENERAdores y CUOTAS DE MERCADO}

Con un valor de mercado mundial estimado a exceder los 170 mil millones de USD en el año 2024 [8], y a medida que el mercado de la energía eólica ha ido madurando, cada vez se hace más difícil encontrar información gratuita actualizada sobre el tema, sin embargo varias firmas de consultoría y análisis de mercado ponen a disposición del público información tan reciente como 2017, donde los primeros cuatro principales suministradores de turbinas eólicas en el mundo fueron la danesa Vestas con $16.7 \%$, la unión alemana española Siemens Gamesa con $16.6 \%$, la china Goldwind con $10.5 \%$ y la estadounidense GE Renewable con $7.6 \%$ de un total contabilizado de 52,150 MW, y para América Latina los primeros cuatro fueron GE Renewable con $26.57 \%$, Siemens Gamesa con $25.48 \%$, Vestas con $22.15 \%$ y Nordex Acciona con $11.9 \%$ de 2,905 MW [9]. En este mismo informe se reporta que Siemens Gamesa contaba con el $59.3 \%$ de la capacidad instalada acumulada total costa afuera en el mundo, seguido por MHI-Vestas con 16.9\% de 19,295 MW según [9]. 


\section{A. Estructuras}

Existen diferentes tipos de estructuras metálicas que sostienen las turbinas eólicas. Las más representativas de la variedad son las instaladas costa afuera, donde en aguas someras se pueden fijar al lecho marino y en aguas profundas se colocan estructuras flotantes. Las estructuras típicas en aguas poco profundas son llamadas high-rise pile cap, monopile, concrete gravity base, tripod, tri-pile, jacket y suction bucket por sus nombres técnicos en Inglés [2], y las estructuras típicas flotantes son las Spar-buoy, semi-submersible y tension-leg platform también por sus nombres técnicos en Inglés [10].

\section{B. Transmisiones}

Las transmisiones que sirven para unir mecánicamente las aspas de la turbina eólica con su generador eléctrico se pueden catalogar en seis tipos, dos de los cuales no fueron utilizados en 2017, que fueron la Full Hydraulic y la Hybrid Hydraulic. El tipo de transmisión más común, con el $68.8 \%$ del mercado es la del tipo Conventional Drive, en segundo lugar con $21.6 \%$ se encuentra la PMG Direct Drive, en tercero la EESG Direct Drive con $6.8 \%$ y el cuarto lugar la del tipo Hybrid Drive con $2.9 \%[9]$.

\section{Generadores}

Los cuatro tipos de generadores que se utilizan para la generación de energía eólica a gran escala son el generador de inducción doblemente alimentado (DFIG por sus siglas en Inglés), el generador de inducción de jaula de ardilla (SCIG por sus siglas en Inglés), el generador sincrónico excitado eléctricamente (EESG por sus siglas en Inglés) y el generador sincrónico de imanes permanentes (PMSG por sus siglas en Inglés). En 2014 se realizó una proyección de la participación en el mercado de cada uno de estos tipos de generadores hasta el 2018 como se reporta en [11], donde poco a poco ha ido aumentando la participación en el mercado de los PMSG hasta llegar a un $31.04 \%$, los EESG con un $10.1 \%$, los SCIG con $8.46 \%$ y los DFIG con $50.4 \%$. En este rubro los cinco principales fabricantes en 2018 fueron ABB, Suzlon, SANY, Nordex y GE Renewable [12].

\section{Convertidores}

Los convertidores electrónicos de potencia utilizados en la energía eólica se pueden clasificar en cuatro tipos, los de Pequeña Escala, Escala Comunitaria, Escala de Empresa de Suministro de Energía Costa Adentro y la Escala de Empresa de Suministro de Energía Costa Afuera. En 2014 también se realizó una proyección de la participación en el mercado de cada una de estas categorías hasta 2018 como se reporta en [13], donde también poco a poco ha ido aumentando la participación en el mercado de los convertidores para uso costa afuera hasta llegar a $12.55 \%$ en 2018 , los de costa adentro de escala de empresa de suministro de servicios de energía con $66.4 \%$ de participación, los de escala comunitaria con $18.21 \%$ y los de pequeña escala con $2.84 \%$. En este rubro los cinco principales fabricantes en 2018 fueron Sungrow Power Supply, Corona, Schneider, Emerson Network Power y ABB [14].

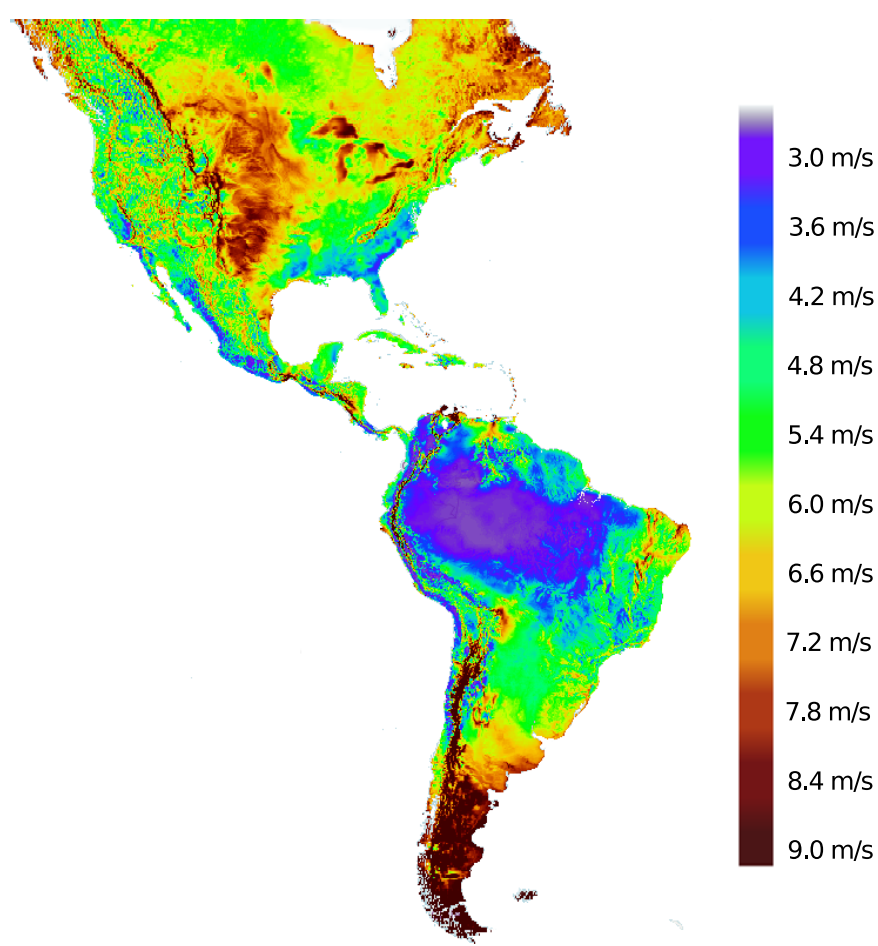

Figura 3. Atlas global para energías renovables - Opción Viento [15].

\section{Metas gubernamentales de Generación DE ENERGÍA RENOVABLE}

Con el objetivo de facilitar el acceso a la información de mapas mundiales de energías renovables, sistemas eléctricos de la mayoría de las naciones, estudios de factibilidad de interconexión a la red eléctrica, y demás documentación, para proporcionar información de inteligencia para incentivar y atraer la inversión en generación de electricidad con recursos renovables, la Agencia Internacional de Energía Renovable (IRENA por su siglas en Inglés) pone a disposición de forma gratuita un sin número de reportes e información consultable en línea. Como un ejemplo de esta información se presenta en la Fig. 3 un mapa del potencial eólico en el continente americano.

Asimismo, la IEA también suministra una serie de reportes y estadísticas hasta el 2016 donde por ejemplo, la generación de electricidad con energía eólica en el mundo fue del $3.8 \%$ del total y ésta a su vez, representó el $16.7 \%$ del total aportado por las energías renovables [16].

Después de llevarse a cabo el Acuerdo de París en 2015, la mayoría de las naciones del mundo se pusieron de acuerdo para establecer objetivos de energía renovable [17] y eficiencia energética [18] para poco a poco dejar de emitir gases de efecto invernadero a la atmósfera y convertirse en sociedades de bajas emisiones. A continuación se ejemplifican las políticas energéticas de tres países, dos de América Latina representando mercados energéticos emergentes y uno de Europa representando un mercado eléctrico consolidado.

\section{A. Dinamarca}

Este país de primer mundo está declarado como el primer país desarrollado que será independiente de los combustibles 
fósiles con la meta de utilizar un $100 \%$ de energía renovable en el año 2050 [19]. Esta meta le ha valido ser el estandarte de campañas de utilización de energía renovable de la organización Greenpeace [20], y la misma Agencia de Energía Danesa, en su pronóstico de energía y clima 2018 anuncia la necesidad de nuevas iniciativas para llevar a cabo sus metas ya que de seguir con las mismas políticas actuales el consumo de energía fósil continuará bajando, pero a partir de 2022 se aumentaría de nuevo el consumo de carbón por lo que desde ahora mismo se advierte y se pone a disposición del mundo toda la información al respecto para que se lleguen a buenos resultados en las negociaciones con la unión europea rumbo al cumplimiento de los objetivos de reducción de emisiones en conjunto [21]. En 2016, la energía eólica tuvo una participación del $41.9 \%$ del total de energía eléctrica generada en el país, y sumando el resto de las energías renovables se obtuvo una participación del $62.8 \%$ [16].

\section{B. México}

De acuerdo con los datos reportados en el Programa Especial para el Aprovechamiento de las Energías Renovables 2014-2018 [22] de la Secretaría de Energía del gobierno de México, las metas decretadas a nivel de Ley para el Aprovechamiento de las Energías Renovables y el Financiamiento de la Transición Energética, son de $35 \%$ de la generación anual de electricidad con energías limpias para 2024, $40 \%$ para 2035 y del $50 \%$ para 2050. En el Reporte de Avance de Energías Limpias del Primer Semestre 2018 [23], también de la Secretaría de Energía del gobierno de México, se visualiza que el resultado de un $24.12 \%$ del total de energía eléctrica generada fue con energías limpias, de las cuales $17.29 \%$ fueron renovables, con la energía eólica con una participación del $3.63 \%$. La capacidad instalada de las energías renovables representa el $27.09 \%$ del total en el país.

\section{Chile}

Con base en la política energética de Chile, llamada Energía 2050 [24], los principales objetivos para 2035 y 2050 que ayudarán a cumplir las metas climáticas son aquellos sobre el uso de energías renovables en la generación de electricidad (incrementando a $60 \%$ la participación en 2035 y a $70 \%$ en 2050), la promoción del uso de combustibles de bajas emisiones y la adopción de una ley de eficiencia energética. De acuerdo con los datos publicados en el Reporte Mensual del Sector Energético de Agosto 2019, por parte de la Comisión Nacional de Energía de Chile, un $18 \%$ de la generación de energía eléctrica estuvo a cargo de las energías renovables no convencionales, con la energía eólica con una participación del $7 \%$ [25]. La capacidad instalada de las energías renovables para entonces fue del $21.5 \%$ del total en el país [26]. Un mapa de viento y demás datos relacionados se encuentran disponibles en [27] por iniciativa del Ministerio de Energía del gobierno de Chile.

Estos son tres ejemplos con situaciones particulares específicas. Aún y cuando no todos los países del mundo se encuentren alineados con los objetivos del Acuerdo de París, no quiere decir que estén produciendo su energía sin utilizar recursos renovables. En [28] se puede constatar que existen países que actualmente producen su energía eléctrica con recursos $100 \%$ renovables, como Paraguay, por mencionar un ejemplo de América Latina.

\section{ADOPCIÓN DE ENERGÍAS RENOVABLES EN LA INDUSTRIA DEL GAS Y DEL PETRÓLEO}

Existe una proyección del avance mundial en el uso de energías renovables, publicada en el documento de la IRENA [29], donde se hace notar el comienzo del crecimiento exponencial en el uso de energías renovables en el año 2015, el pico mundial de la demanda de combustibles fósiles en 2025 , donde las energías renovables sobrepasan a las fósiles en 2050 y donde para el año 2090 ya nos habremos convertido en una sociedad de bajas emisiones afinando detalles para cumplir con creces los objetivos y metas del Acuerdo de París. Esta proyección es tomada del Sky Scenario 2018 de la compañía Shell.

Datos hechos públicos por la firma de consultoría y análisis de mercado Lazard en [30], confirman lo que las compañías petroleras saben desde hace muchos años, que invertir en energías renovables es un buen negocio, con precios nivelados de la energía muy competitivos por parte de la energía eólica y solar. Con esto se han planteado entonces pasar de ser compañías proveedoras de combustibles fósiles a convertirse en compañías proveedoras de energía. Dos ejemplos enunciativos son las compañías BP y Shell, quienes tienen amplias inversiones en parques eólicos costa adentro y costa afuera, y quienes aprovechan los recursos renovables para generación de electricidad, calentamiento y enfriamiento, fuerza motriz y hasta para producir biocombustibles [31]. No obstante, muchas otras compañías han comenzado ya su transformación para dejar de ser espectadores y convertirse en actores y partícipes de la transición energética donde la energía eólica se perfila para tener una participación de más de una cuarta parte de la generación de electricidad en el mundo en el largo plazo.

\section{CONCLUSIONES}

Se ha presentado que el año 2015 ha sido el mejor año hasta finales de 2018 en cuanto a nuevas instalaciones, pero, con 185 países que ya han ratificado el Acuerdo de París de 197 participantes en la convención, y esperando que el mercado eólico siga con una razón de crecimiento anual compuesta del $2.7 \%$ hasta 2023, incluso mejores años están por venir. Brasil, Chile, Argentina y México impulsan a Latinoamérica junto con la contribución de otros mercados más pequeños. La energía eólica costa afuera se encuentra siguiendo la curva de costos hacia abajo de la costa adentro; sin embargo, a la costa adentro le tomó 30 años llegar a precios realmente competitivos. Para la energía eólica en su conjunto, los impulsores del mercado siguen en su lugar, y cada vez más prominentes: la seguridad energética; estabilidad de costos; seguridad macroeconómica; desarrollo económico local y creación de empleo; medio ambiente y clima local (descarbonización).

\section{AGRADECIMIENTOS}

Este trabajo fue financiado por el Proyecto Regular Fondecyt 1160690. 


\section{REFERENCIAS}

[1] IRENA, "Global energy transformation: A roadmap to 2050 (2019 edition)," International Renewable Energy Agency, Abu Dhabi, Tech. Rep. ISBN 978-92-9260-121-8, Apr. 2019. [Online]. Available: https://www.irena.org/-/media/Files/IRENA/Agency/Publication/2019/A pr/IRENA_Global_Energy_Transformation_2019.pdf

[2] IEA-ETSAP and IRENA, "Wind Power Technology Brief," International Renewable Energy Agency, Abu Dhabi, Tech. Rep., Mar. 2016. [Online]. Available: https://www.irena.org/-/media/Files/IRENA/Agency/Publicat ion/2016/IRENA-ETSAP_Tech_Brief_Wind_Power_E07.pdf

[3] MHI Vestas. (2018) Leading edge technology. Turbines and Innovations. MHI Vestas Offshore Wind A/S, Dusager 4, 8200 Aarhus N, Denmark. [Online]. Available: http://www.mhivestasoffshore.com/innovations/

[4] GE Renewable Energy. (2019) HALIADE-X OFFSHORE WIND TURBINE PLATFORM. General Electric Renewable Energy's offshore wind business headquarters and engineering office, Nantes, France. [Online]. Available: https://www.ge.com/renewableenergy/windenergy/offshore-wind/haliade-X-offshore-turbine

[5] M. Morris. (2018, Sep.) MHI Vestas Launches the First 10 MW Wind Turbine in History. MHI Vestas Offshore Wind A/S, Dusager 4, 8200 Aarhus N, Denmark. [Online]. Available: http://www.mhivestasoffshore .com/mhi-vestas-launches-the-first-10-mw-wind-turbine-in-history/

[6] K. Ohlenforst, S. Sawyer, A. Dutton, B. Backwell, R. Fiestas, J. Lee, L. Qiao, F. Zhao, and N. Balachandran, "GWEC । GLOBAL WIND REPORT 2018," Global Wind Energy Council, Rue d'Arlon 80, 1040 Brussels, Belgium, Tech. Rep., Apr. 2019. [Online]. Available: https://gwec.net/global-wind-report-2018/

[7] L. Fried, S. Shukla, S. Sawyer, and S. Teske, "GLOBAL WIND ENERGY OUTLOOK I 2016," Global Wind Energy Council, Rue d'Arlon 80, 1040 Brussels, Belgium, Tech. Rep., Oct. 2016. [Online]. Available: http://files.gwec.net/register?file=/files/GlobalWindEnergyO utlook2016

[8] Global Market Insights. (2018, Jul.) Offshore Wind Energy Market Report 2018-2024. 4 North Main Street, Selbyville, Delaware 19975 USA. [Online]. Available: https://www.windpowerengineering.com /business-news-projects/uncategorized/offshore-wind-energy-marketexpected-to-exceed-usd-60-billion-by-2024/

[9] F. Zhao, A. Karcanias, R. Clover, C. LeWand, and G. Ramakrishnan, "Global Wind Market Update - Demand \& Supply 2017 Part One Supply Side Analysis," FTI Consulting, Gl. Kongevej 1, Copenhagen 1630, Denmark, Tech. Rep., Apr. 2018. [Online]. Available: https:// www.fticonsulting.com/ /media/Files/us-files/intelligence/intelligenceresearch/global-wind-market-update-2017-supply-side-analysis.pdf

[10] IRENA, "Innovation Outlook: Offshore Wind," International Renewable Energy Agency, Abu Dhabi, Tech. Rep. ISBN 978-92-95111-36-3, Oct. 2016. [Online]. Available: https://www.irena.org/DocumentDownloads/ Publications/IRENA_Innovation_Outlook_Offshore_Wind_2016.pdf

[11] J. Tang, "The World Market for Wind Generators," IHS Technology, Englewood, Colorado, USA, Abstract, Oct. 2014. [Online]. Available: https://technology.ihs.com/api/binary/518987

[12] HTF Market Intelligence Consulting. (2019, Apr.) 2013-2028 Report on Global Wind Turbine Generator Market by Player, Region, Type, Application and Sales Channel. Unit No. 429, Parsonage Road Edison, New Jersey USA - 08837. [Online]. Available: https: //asiancrunch.org/wind-turbine-generator-market-astonishing-growthin-coming-yearscenturion-energy-alternative-energy-intechopen/34433/

[13] J. Tang, "The World Market for Wind Converters," IHS Technology, Englewood, Colorado, USA, Abstract, Oct. 2014. [Online]. Available: https://technology.ihs.com/api/binary/518988

[14] HTF Market Intelligence Consulting. (2019, Apr.) Global Wind Power Converter Market Analysis 2013-2018 and Forecast 2019-2024. Unit No. 429, Parsonage Road Edison, New Jersey USA - 08837. [Online] Available: https://majoreports.com/wind-power-converter-market-isbooming-worldwidexin-fengguang-electronic-shandong-bos-powersupply-shenzhen-hopewind-electric/51163/

[15] IRENA. (2019) Global Atlas for Renewable Energy - VAISALA Global Wind Dataset $5 \mathrm{~km}$ onshore wind speed at $80 \mathrm{~m}$ height units in $\mathrm{m} / \mathrm{s}$. Abu Dhabi. [Online]. Available: https://irena.masdar.ac.ae/gallery/\#map/543

[16] IEA. (2019) Statistics data browser - Global energy data at your fingertips. International Energy Agency, 31-35 rue de la Fédération, 75739 Paris Cedex 15, France. [Online]. Available: https://www.iea.org/statistics/

[17] IRENA, "Renewable Energy Target Setting," International Renewable Energy Agency, Masdar City, P.O. Box 236, Abu Dhabi, United Arab
Emirates, Tech. Rep. ISBN 978-92-95111-69-1, Jun. 2015. [Online]. Available: https://www.irena.org/DocumentDownloads/Publications/IRE NA_RE_Target_Setting_2015.pdf

[18] REN21, "Renewables 2018 Global Status Report," Renewable Energy Policy Network for the 21st Century, c/o UNEP DTIE, Building VII, 1 rue Miollis, 75015 Paris France, Tech. Rep. ISBN 978-3-9818911-3-3, Jun. 2018. [Online]. Available: http://www.ren21.net/wp-content/upload s/2018/06/17-8652_GSR2018_FullReport_web_final_.pdf

[19] Danish Ministry of Energy, Utilities and Climate. (2019) Danish climate policies. The Danish Energy Agency, Carsten Niebuhrs Gade 43, DK-1577 Copenhagen V, Denmark. [Online]. Available: https://ens.dk/en/our-responsibilities/energy-climate-politics/danishclimate-policies

[20] Greenpeace, "Denmark's commitment to $100 \%$ renewable energy," Greenpeace International, Ottho Heldringstraat 5, 1066 AZ Amsterdam, The Netherlands, Greenpeace \& WFC $100 \%$ RE campaign briefing, Oct. 2014. [Online]. Available: http://www.go100re.net/wp-content/uplo ads/2014/10/GP-and-WFC_Denmarks-commitment-to-100-renewableenergy.pdf

[21] Danish Energy Agency, "Denmark's Energy and Climate Outlook 2018: Baseline Scenario Projection Towards 2030 With Existing Measures (Frozen Policy)," The Danish Energy Agency, Amaliegade 44, 1256 Copenhagen K, Denmark, Tech. Rep. ISBN 978-87-93180-34-5, Jul. 2018. [Online]. Available: https://ens.dk/sites/ens.dk/files/Basisfremskri vning/deco18.pdf

[22] Secretaría de Energía, "Programa Especial para el Aprovechamiento de las Energías Renovables 2014-2018," Secretaría de Energía de los Estados Unidos Mexicanos, Insurgentes Sur 890, Del Valle, Ciudad de México. C.P. 03100, México, Tech. Rep., Mar. 2014. [Online]. Available: https://www.gob.mx/cms/uploads/attachment/file/249/PEAER-2014.pdf

[23] — , "Reporte de Avance de Energías Limpias Primer Semestre 2018," Secretaría de Energía de los Estados Unidos Mexicanos, Insurgentes Sur 890, Del Valle, Ciudad de México. C.P. 03100, México, Tech. Rep., Nov. 2018. [Online]. Available: https://www.gob.mx/cms/uploads /attachment/file/418391/RAEL_Primer_Semestre_2018.pdf

[24] Ministerio de Energía, "Energy 2050: Chile's Energy Policy,” Ministerio de Energía, Gobierno de Chile, Alameda \#1449, Edificio Santiago Downtown II, Pisos 13 y 14, Santiago, Chile, Tech. Rep., Aug. 2016. [Online]. Available: http://www.energia2050.cl/wp-content/uploads/20 16/08/Energy-2050-Chile-s-Energy-Policy.pdf

[25] Comisión Nacional de Energía, "Reporte Mensual Sector Energético Agosto 2019," Ministerio de Energía, Gobierno de Chile, Avenida Libertador Bernardo O'Higgins 1449, Edificio Santiago DownTown, Torre 4, Piso 13, Santiago Centro, Chile, Tech. Rep. Vol. $N^{\circ} 54$, Aug. 2019. [Online]. Available: https://www.cne.cl/wp-content/uploads/2019 /08/RMensual_v201908.pdf

[26] — - "Reporte Mensual ERNC Agosto 2019," Ministerio de Energía, Gobierno de Chile, Avenida Libertador Bernardo O’Higgins 1449, Edificio Santiago DownTown, Torre 4, Piso 13, Santiago Centro, Chile, Tech. Rep. Vol. N ${ }^{\circ} 36$, Aug. 2019. [Online]. Available: https://www.cn e.cl/wp-content/uploads/2019/08/RMensual_ERNC_v201908.pdf

[27] Ministerio de Energía / GIZ / Universidad de Chile. (2019) Explorador de Energía Eólica. Departamento de Geofísica, Facultad de Ciencias Físicas y Matemáticas, Universidad de Chile, Avenida Blanco Encalada 2002, Santiago, Chile. [Online]. Available: http://walker.dgf.uchile.cl/E xplorador/Eolico2/

[28] IEA. (2019) Statistics - Atlas of Energy - Share of Renewables in Electricity Production. International Energy Agency, 31-35 rue de la Fédération, 75739 Paris Cedex 15, France. [Online]. Available: http://energyatlas.iea.org/\#!/tellmap/-1076250891/4

[29] IRENA, "A New World: The Geopolitics of the Energy Transformation," International Renewable Energy Agency, Masdar City, P.O. Box 236 Abu Dhabi, United Arab Emirates, Tech. Rep. ISBN 978-929260-097-6, Jan. 2019. [Online]. Available: https://www.irena.org//media/Files/IRENA/Agency/Publication/2019/Jan/Global_commissio n_geopolitics_new_world_2019.pdf

[30] Lazard, "Levelized Cost of Energy Analysis, Version 12.0," Lazard, 30 Rockefeller Plaza, New York NY 10112, USA, Tech. Rep., Nov. 2018. [Online]. Available: https://www.lazard.com/media/450784/lazardslevelized-cost-of-energy-version-120-vfinal.pdf

[31] J. Switzer. (2013) When renewables meet the oil and gas industry, opposites attract. The Pembina Institute, Head office, 219 - 19 Street NW, Calgary, AB, T2N 2H9 Canada. [Online]. Available: https://www.pembina.org/blog/when-renewables-meet-oil-and-gasindustry-opposites-attract 